\title{
Magnetic Force Microscopy of Low-Coercivity Ferromagnetic Nanodiscs
}

\author{
Victor L. Mironov ${ }^{1}$, Boris A. Gribkov ${ }^{1}$, Dmitry S. Nikitushkin ${ }^{1}$, Sergey A. Gusev ${ }^{1}$, Sergey V. Gaponov ${ }^{1}$, \\ Andrey B. Shubin ${ }^{2}$, Peter A. Zhdan ${ }^{3}$, and Chris Binns ${ }^{4}$ \\ ${ }^{1}$ Institute for physics of microstructures RAS, Nizhniy Novgorod 603950, Russia \\ 2"Nanotechnology MDT" Company, Zelenograd 124482, Russia \\ ${ }^{3}$ School of Engineering, University of Surrey, Guildford, Surrey GU2 7XH, U.K. \\ ${ }^{4}$ Department of Physics and Astronomy, University of Leicester, Leicester LE1 7RH, U.K.
}

\begin{abstract}
We report the results of magnetic force microscopy (MFM) investigations of low-coercivity Co nanodiscs, with 50 nm lateral size and $20 \mathrm{~nm}$ height, fabricated by e-beam lithography and ion etching. We observed two types of MFM contrast in the form of Gaussian and ring distributions caused by strong probe-particle interaction. We compared experimentally the transformation of the MFM contrast from these low-coercivity nanodiscs caused by an external magnetic field applied in situ, and compared the experimental results with theoretical simulations.
\end{abstract}

Index Terms-Low-coercivity magnetic nanodiscs, magnetic force microscopy, tip-sample interaction.

\section{INTRODUCTION}

$\mathbf{I}$ NVESTIGATIONS of magnetic states in small ferromagnetic nanoparticles by magnetic force microscopy (MFM) methods have a great importance both from fundamental and practical points of view, especially in connection with the development of high-density magnetic data storage systems [1], [2]. Reducing the lateral sizes of particles down to $50 \mathrm{~nm}$ leads to the formation of single-domain states and in the absence of internal anisotropy, the coercivity of these particles becomes comparable with the MFM probe field. In this case MFM contrast is formed in the presence of a strong interaction between the probe and the particles, which leads to difficulties in the interpretation of experimental MFM images [3]-[6]. Recently, we considered theoretically the possibility of using an external magnetic field to stabilize the magnetic moment in small, low-coercivity (LC) nanoparticles [7]. Here we report the results of experimental investigations of the MFM contrast peculiarities from LC ferromagnetic Co nanodiscs in an external magnetic field, which confirm the previous calculations.

\section{EXPERIMENTAL TECHNIQUE}

The ordered arrays of ferromagnetic nanodiscs were fabricated by e-beam lithography and subsequent ion etching of thin Co films [8]. The particle thickness was $20 \mathrm{~nm}$ and the lateral size varied in the range $50-70 \mathrm{~nm}$. The electron microscopy and e-beam lithography were performed using a "JEOL-JEM 2000EX II" scanning electron microscope. The investigations of the discs' magnetic states were carried out using a vacuum scanning probe microscope, "Solver HV," manufactured by "NTMDT" Company. This was equipped with a dc electromagnet incorporated in the vacuum vibroisolating platform. MFM measurements were performed in the oscillatory noncontact (con-

Digital Object Identifier 10.1109/TMAG.2008.2000811

Color versions of one or more of the figures in this paper are available online at http://ieeexplore.iee.org.

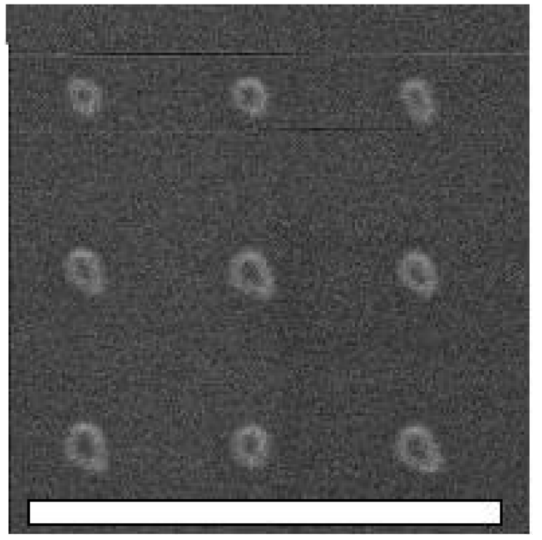

Fig. 1. SEM image of Co nanodisc array (plan view in secondary electrons). The white scale bar is $1 \mu \mathrm{m}$.

stant height) mode using cantilevers with $50 \mathrm{~nm}$ Co coating. The double-amplitude of cantilever oscillations was about $30 \mathrm{~nm}$ and the average scanning height was $50-60 \mathrm{~nm}$. The phase shift of cantilever oscillations caused by the gradient of the magnetic force was registered as the MFM contrast. All measurements were performed in a vacuum of $10^{-4}$ torr, which increases the MFM signal due to an increase in the cantilever quality factor.

\section{MODELING OF MFM CONTRAST FOR LOW-COERCIVITY FERROMAGNETIC PARTICLES}

The point probe approximation [9] was used in the model calculations. The MFM tip was approximated as a uniformly magnetized sphere with an effective magnetic moment, $m_{t}=M_{t} V_{t}$ $\left(M_{t}\right.$ is the remanent magnetization of the tip capping material and $V_{t}$ is the effective volume of the interactive part of the tip). Analysis of experimental data shows that the typical effective volume of magnetic material for our probes with Co coating $\left(M_{t} \sim 1400 \mathrm{G}\right)$ is approximately $2 \cdot 10^{5} \mathrm{~nm}^{3}$. The distribution of phase shift, $\Delta \varphi$, for cantilever oscillations was calculated as a model MFM contrast: 


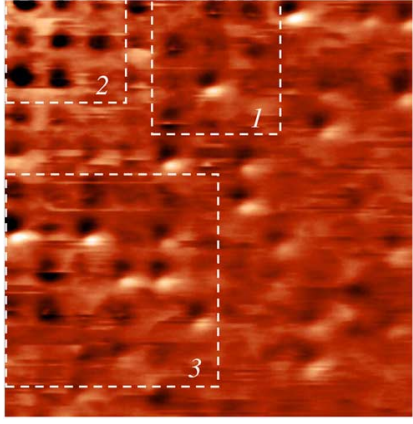

Fig. 2. MFM image of Co particles array. Frame size is $3.5 \times 3.5 \mu \mathrm{m}$. The characteristic areas are marked by squares and numbers.
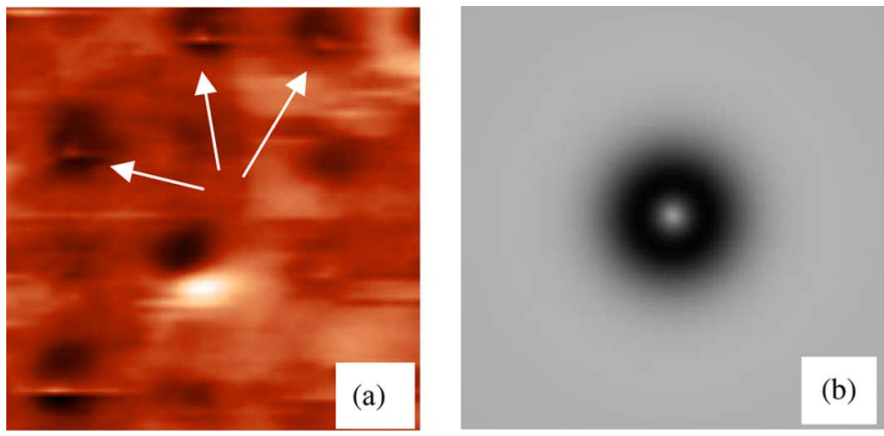

Fig. 3. (a) Experimental circle distributions of MFM contrast from LC discs (indicated by white arrows). Frame size is $1.2 \times 1.2 \mu \mathrm{m}$. (b) Simulated circle MFM contrast.

where $Q$ is the cantilever quality factor, $K$ is the cantilever force constant, and $F_{z}$ is the $z$-component of the force. The calculations of model MFM images were performed in the dipole-dipole approximation. The details of computer simulations of peculiarities in MFM contrast formation for LC nanoparticles are reported in [7].

\section{RESUlTS AND DiscUSSIONS}

A typical SEM image (plan view in secondary electrons) of fabricated Co nanodiscs is represented in Fig. 1. The SEM contrast in this mode underscores the discs' edges [10]. The discs vary in lateral size (50-70 $\mathrm{nm}$ ) and shape (circles and ellipses).

The constant height mode MFM image of the fabricated array of Co nanodiscs is represented in Fig. 2. The preliminary sample magnetization was zero. In one experiment, we tilted the sample to obtain different tip-sample separations. The inclination was set by means of stays regulation and was checked in atomic force microscopy mode. Thus, during MFM imaging the scanning height was reduced from $70 \mathrm{~nm}$ at the bottom right corner (Fig. 2) to $30 \mathrm{~nm}$ at the top left corner, so the strongest tip-particle interaction was observed in the area 2 in Fig. 2. Note, that only some of the discs with an elliptical shape demonstrate bipolar MFM contrast, which corresponds to a uniform magnetization in the sample plane. Most discs show a complicated MFM contrast distribution.

The MFM image that demonstrates the MFM contrast typical for LC discs with their magnetic moment confined in the disc plane (area 1 in Fig. 2) is presented in Fig. 3(a). During scanning
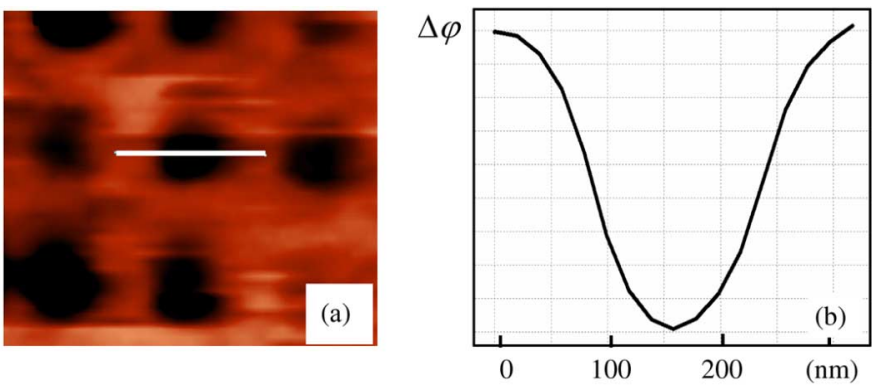

Fig. 4. (a) Experimental Gaussian distribution of MFM contrast from LC discs. Frame size is $1.2 \times 1 \mu \mathrm{m}$. (b) Profile of the contrast distribution (arb. units) along the white line.
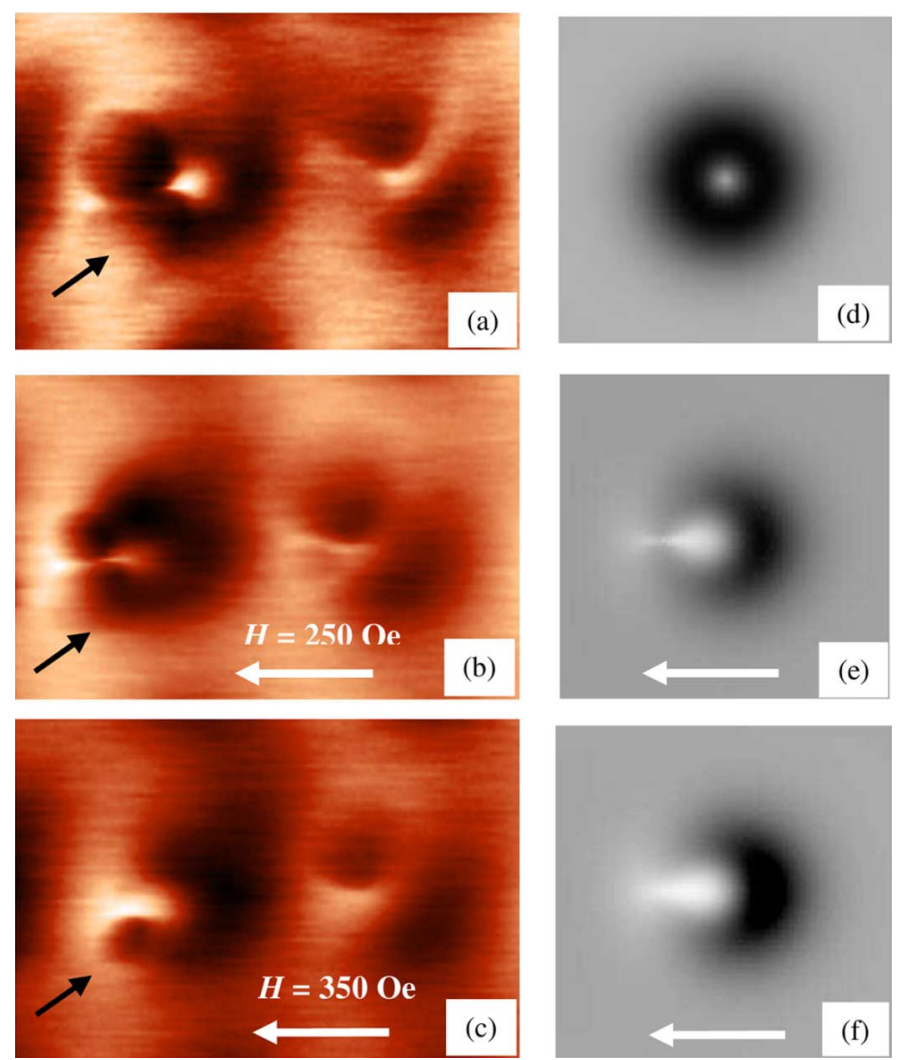

Fig. 5. Evolution of the ring contrast from LC disc (indicated by black arrows) in an external magnetic field. (a) External magnetic field, $H=0$. (b) $H=$ 250 Oe. (c) $H=350$ Oe. The frame size is $450 \times 300 \mathrm{~nm}$. (d)-(f) are corresponding model MFM images. The external field direction is indicated by white arrows.

in area 1 , the magnetic field from the MFM tip, $H_{t}$, became larger than the coercive field, $H_{c}$, of the discs, $H_{t}>H_{c}$, and the contrast distributions had a ring shape (marked by arrows), which corresponds to the rotation of the disc magnetic moment in the magnetic field of the scanning tip. For comparison, the model MFM distribution predicted in [7] is shown in Fig. 3(b).

When the distance between the probe and the particle was further reduced (area 2 in Fig. 2), the probe-particle interaction increased, $H_{t}>>H_{c}$, and the MFM contrast distribution became Gaussian (Fig. 4), which corresponds to the out-of-disc plane magnetization disturbance. In this case, the magnetization of the disc is fully oriented along the probe field during scanning. 

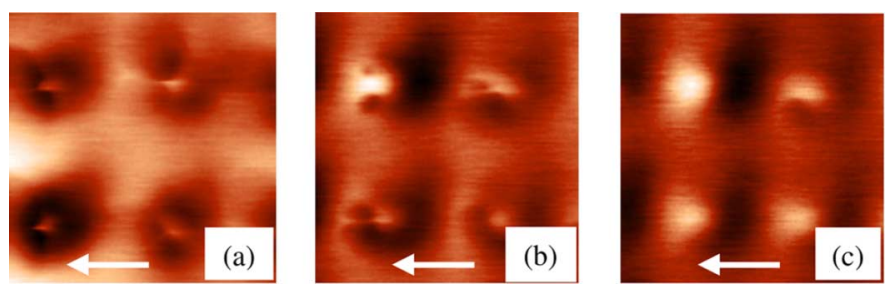

Fig. 6. Redistribution of the ring contrast under external magnetic field. (a) Magnetic field $H=0$. (b) $H=300 \mathrm{Oe}$. (c) $H=360$ Oe. The frame size is $450 \times 450 \mathrm{~nm}$. The external field direction is indicated by white arrows.
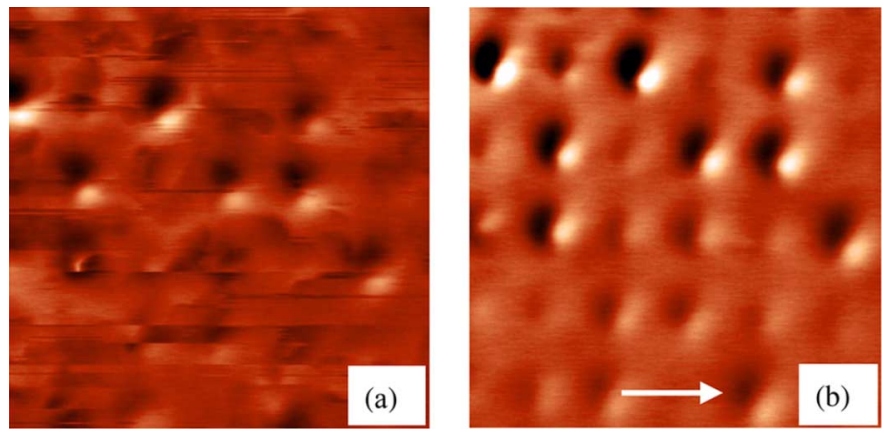

Fig. 7. Stabilization of the MFM contrast in the external field. (a) Enlarged image of area 3 in Fig. 2. (b) MFM image of the same area with an applied external field of about $350 \mathrm{Oe}$ (white arrow indicates the external field direction). The frame size is $2 \times 2 \mu \mathrm{m}$.

The transformations of the ring contrast distribution caused by an external magnetic field were also investigated. Magnetic field, $H_{\mathrm{ex}}$, in the range of \pm 600 Oe was applied in the sample plane. The results of in situ MFM measurements and corresponding computer calculations of the ring contrast evolution due to the external magnetic field are presented in Fig. 5(a)-(f).

The different phases of the bipolar contrast formation predicted in [7] are clearly seen. With increasing external field, $H_{\text {ex }}$, full stabilization of the MFM images was observed. Fig. 6 demonstrates the transformation of the ring contrast caused by increasing $H_{\mathrm{ex}}$. In a strong magnetic field, $H_{\mathrm{ex}}>H_{t}$, the MFM contrast distribution has bright and dark poles [Fig. 6(c)], which correspond to the image of the particle uniformly magnetized along $\vec{H}_{\text {ex }}$.

The same effects of the stabilization of the MFM contrast in an external field were observed for all nanodiscs in different places of the sample (see Fig. 7).

\section{CONCLUSION}

The magnetic force microscopy investigations of low-coercivity Co nanodiscs with lateral sizes $50-70 \mathrm{~nm}$ and $20 \mathrm{~nm}$ height fabricated by e-beam lithography and ion etching were carried out. Two types of MFM contrast in the form of Gaussian and ring distributions caused by strong probe-particle interaction were registered. The Gaussian distribution was observed for low scanning heights. This type of contrast is formed due to full orientation of the sample magnetization along the strong magnetic field of the MFM tip. The ring distribution was observed for the middle scanning heights when the probe field is less than the coercive force, which confines the magnetization of the discs in-plane.

The transformation of the MFM contrast from low-coercivity nanodiscs due to an external magnetic field applied in situ was investigated experimentally. It was shown that the external magnetic field applied in the sample plane stabilizes the magnetic moment of the nanodiscs. When the external field is larger than the tip field, full particle stabilization is observed and MFM contrast distribution has a simple symmetry with bright and dark poles, which corresponds to the image from the particle uniformly magnetized along the external field. It allows selection of the contribution from magnetic tip-sample interaction in the phase MFM contrast. In particular, for complicated samples the external field assisted MFM measurements enable the separation of Van-der-Waals artifacts and real MFM contrast.

\section{ACKNOWLEDGMENT}

The authors are very thankful to S. N. Vdovichev, A. Yu. Klimov, V. V. Rogov for the sample preparation and to Prof. A. A. Fraerman for the very useful discussions. This work was supported in part by RFBR (08-02-01202) and by EC through the NANOSPIN project (contract NMP4-CT-2004-013545).

\section{REFERENCES}

[1] J. I. Martin, J. Nogues, K. Liu, J. L. Vicent, and I. K. Schuller, "Ordered magnetic nanostructures: fabrication and properties," J. Magn. Magn. Mater., vol. 256, pp. 449-501, 2003.

[2] A. Kikitsu, Y. Kamata, M. Sakurai, and K. Naito, "Recent progress of patterned media," IEEE Trans. Magn., vol. 43, no. 9, pp. 3685-3688, Sep. 2007.

[3] M. Rasa and A. P. Philipse, "Scanning probe microscopy on magnetic colloidal particles," J. Magn. Magn. Mater., vol. 252, pp. 101-103, 2002.

[4] M. Rasa, B. W. M. Kuipers, and A. P. Philipse, "Atomic force microscopy and magnetic force microscopy study of model colloids," $J$. Colloid Interface Sci., vol. 250, pp. 303-315, 2002.

[5] S. A. Koch, R. H. t. Velde, G. Palasantzas, and J. T. M. D. Hosson, "Magnetic versus structural properties of Co nanocluster thin films: A magnetic force microscopy study," Appl. Phys. Lett., vol. 84, no. 4, pp. 556-558, Jan. 2004.

[6] S. A. Koch, R. H. t. Velde, G. Palasantzas, and J. T. M. D. Hosson, "Magnetic force microscopy on cobalt nanocluster films," Appl. Surf. Sci, vol. 226, pp. 185-190, 2004.

[7] V. L. Mironov, D. S. Nikitushkin, C. Binns, A. B. Shubin, and P. A. Zhdan, "Magnetic force microscope contrast simulation for low-coercive ferromagnetic and superparamagnetic nanoparticles in an external magnetic field," IEEE Trans. Magn., vol. 43, no. 11, pp. 3961-3963, Nov. 2007.

[8] A. A. Fraerman, S. A. Gusev, L. A. Mazo, I. M. Nefedov, Yu. N. Nozdrin, I. R. Karetnikova, M. V. Sapozhnikov, I. A. Shereshevsky, and L. V. Sukchodoev, "Rectangular lattices of permalloy nanoparticles: Interplay of single-particle magnetization distribution and interparticle interaction," Phys. Rev. B, vol. 65, pp. 064424 1-064424 5, 2002.

[9] U. Hartmann, "The point dipole approximation in magnetic force microscopy," Phys. Lett. A, vol. 137, pp. 475-479, 1989.

[10] J. Goldstein, D. Newbury, P. Echlin, D. Joy, C. Fiori, and E. Lifshin, Scanning Electron Microscopy and X-ray Microanalysis. New York: Plenum, 1981.

Manuscript received October 22, 2007; revised May 14, 2008. Current version published September 19, 2008. Corresponding author: V. L. Mironov (e-mail: mironov@ipm.sci-nnov.ru). 